\title{
Microstructural and Hardness Behavior of AlCoCuFeNi and AlCoCuFeNiCr High- Entropy Alloys Synthesized by Milling and Arc Melting
}

\author{
C.D. Gomez-Esparza ${ }^{1}$, M. Hernandez-Hernandez², V.H. Mercado-Lemus ${ }^{2}$, and R. Pérez-Bustamante, ${ }^{2, *}$. \\ 1. Centro de Investigación en Materiales Avanzados (CIMAV), Miguel de Cervantes No.120, C.P. \\ 31109, Chihuahua, Chihuahua., México. \\ 2. CONACYT-Corporación Mexicana de Investigación en Materiales (COMIMSA), Eje 126 225, \\ Industrial San Luis, 78395 San Luis, S.L.P., México. \\ * Corresponding author: raul.perez@comimsa.com
}

High entropy alloys (HEAs) are a new generation of materials containing at least five principal elements in equiatomic or near-equiatomic proportions. The concept behind the development of these alloys is that the entropy is maximum at equiatomic composition. In these alloys the entropy increases as the number of elements increases making difficult the formation of intermetallics [1]. Though conventionally produced by casting routes, alternative processing routes offers a more precise control in the elemental composition of the HEA alloys, avoiding segregation problems and the formation of amorphous phases; granting however, a homogeneous chemical distribution and extending the solid solubility limits imposed in casting processes and with a fraction of the cost [2]. Their attractive physical and mechanical properties attracts the attention of different research groups due to their potential use in the design and repair of parts and tools for the industry of dies, moulds, automotive and aerospace, which application can be carried out through the additive manufacturing technologies currently available.

Elemental powders with purity higher than $99.5 \%$ were used as raw materials to synthesize the equiatomic AlCoCuFeNi and AlCoCuFeNiCr HEA systems (Fig. 1). The MA was performed in a highenergy ball mill (Simoloyer) for a time of $60 \mathrm{~h}$, under an argon atmosphere. Methanol was used as a process control agent to avoid metal agglomeration. The milled powders cold consolidated into cylindrical samples, then melted by arc under in argon atmosphere to prevent oxidation. The ingots were remelted at least 5 times to improve chemical homogeneity. The microstructural characterization was carried out by scanning electron microscopy, whilst the mechanical behaviour was evaluated by means of Vickes microhardness $(\mu \mathrm{HV})$.

Fig. 2 shows secondary electron SEM micrographs of the morphology and particle size distribution of the mechanically milled alloys. The evolution in the morphology from the unmilled powders shown in Fig. 1 is clearly observed in Fig. 2 after $60 \mathrm{~h}$ of milling. Powders shown in Fig. 2 change the accumulated effect of fracture-welding-fracture from the milling process [3]. It can be observed that the predominance of the welding process is observed in the AlCoCuFeNi HEA systems. On the other hand the addition of a sixth element, observed in the AlCoCuFeNi Cr HEA system, improves the welding stage producing as result an increment in the size of the particles. It can be observed the effect of the $\mathrm{Cr}$ element on the particle size of the milled powders affecting noticeable their size and morphology with variation in the $d(0.5)$ mean size from 7.708 to $22.970 \mu \mathrm{m}$. An increment in the particle size suggests a detriment in the mechanical hardness of the $\mathrm{AlCoCuFeNiCr}$ alloy. However, results from mechanical evaluation indicated hardness values of 211 and $291 \mathrm{HV}$ for the $\mathrm{AlCoCuFeNi}$ and $\mathrm{AlCoCuFeNiCr}$ systems respectively. Even though, the addition of a sixth element induces a change in the particle size, the increment in hardness. The study of these HEA systems through transmission electron microscopy 
is the current research topic of the present authors, in order to provide a deeper understanding of the strengthening mechanisms involved in the HEA alloys reported in this work.

\section{References}

[1] W Ji et al., Intermetallics 56 (2015), p. 24.

[2] S Varalakshmi, et al., Materials Science and Engineering: A 527 (2010), p. 1027.

[3] C Suryanarayana, Journal of Alloys and Compounds 509 (2011), p. S229.

[4] M. Hernandez-Hernandez, V.H. Mercado-Lemus, and R. Pérez-Bustamante are grateful with the program Catedras CONACYT (Project 850) for their support.

Figure 1. SEM micrographs of elemental powders used in the synthesis of the HEA alloys
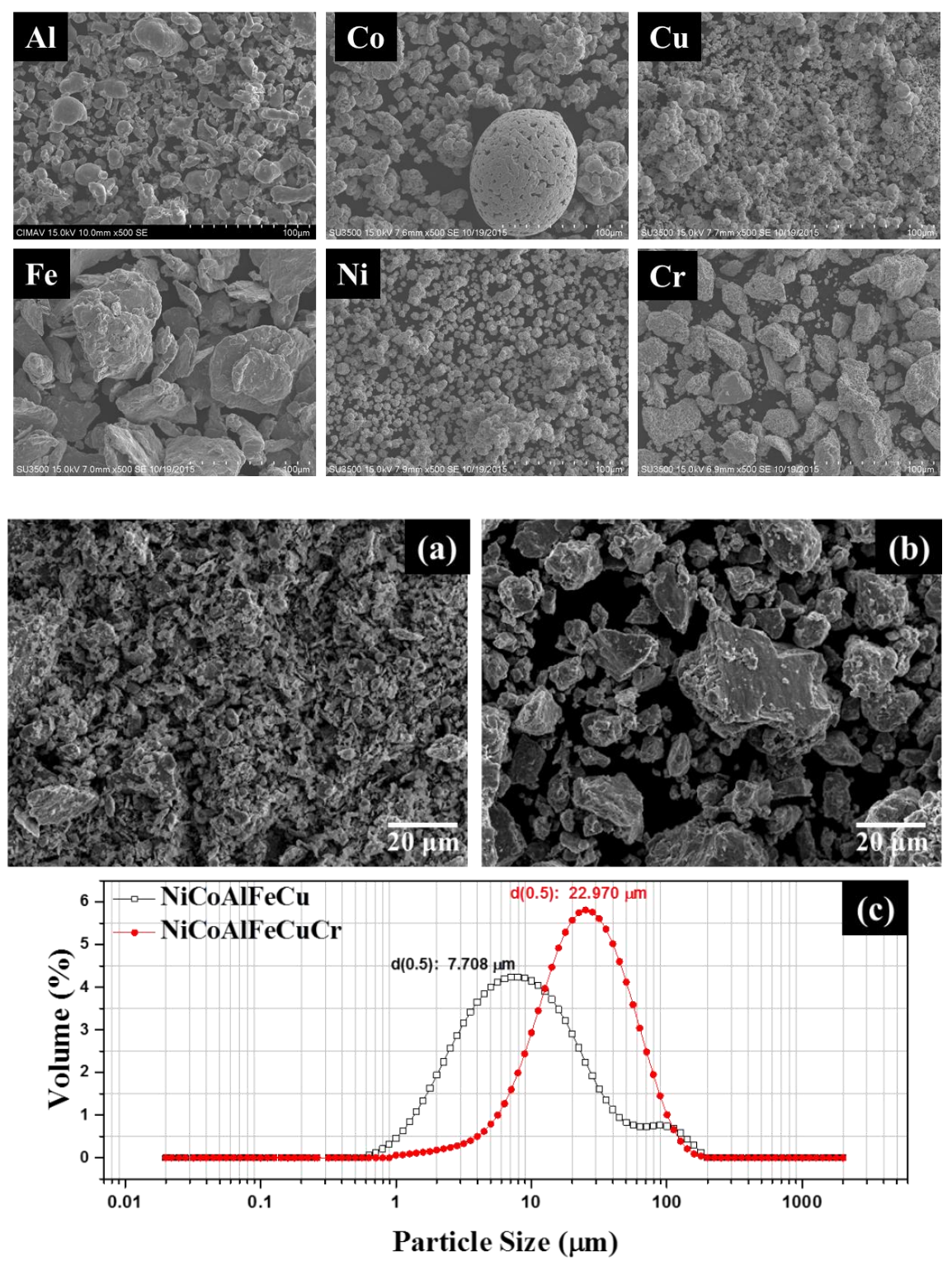

Figure 2. SEM micrographs of powder alloys: (a) AlCoCuFeNi, and (b) AlCoCrCuFeNi showing the morphology after mechanical alloying, and (c) particle size distribution curves. 